ARTIFICIAL SATELLITES, Vol. 46, No. 2 - 2011

DOI: 10.2478/v10018-011-0011-5

\title{
A METHOD TO USE MODIS WATER VAPOR PRODUCTS FOR CORRECTION OF ATMOSPHERIC-INDUCED PHASE IN INTERFEROGRAM
}

\author{
Chaiyapon Keeratikasikorn and Itthi Trisirisatayawong \\ Department of Survey Engineering \\ Chulalongkorn University \\ Bangkok, THAILAND \\ Tel. 66-2-2186662 Fax. 66-2-2186653 \\ Email: chaiyapon@hotmail.com; itthi.t@eng.chula.ac.th
}

\begin{abstract}
DInSAR is a major space-geodetic technique widely used in the study of earth surface deformation. A major source of phase errors in DInSAR technique is heterogeneous phase delay caused mainly by variation of water vapors in troposphere, which is a factor limiting applications of DInSAR mainly to arid areas. This paper presents study results of three methods to correct atmospheric phase errors in DInSAR interferograms formed by TerraSAR-X images. The first method is the use of wet delay derived directly from MODIS precipitable water vapor product. The second method employs ground-based meteorological data to calibrate MODIS PWV before computing phase delays. The third method improves the second method by estimating the expected MODIS PWV value at the time of the TerraSAR-X image acquisitions which over the Bangkok test area is 5 hour earlier than that of MODIS. The time-shifted linear fit model along with the IDW interpolation is used to estimate more realistic phase delays over entire imaging area. From the study of this tropical test area, this time-corrected method provides best results while the second method also achieves a significantly better result than those obtained from direct use of MODIS PWV data.
\end{abstract}

Key words: D-InSAR, Saastamoinen model, MODIS, Zenith wet delay

\section{INTRODUCTION}

Differential Interferometric Synthetic Aperture Radar (DInSAR) is a technique which produces an 'interferogram', an image of phase differences between two SAR images acquired with same viewing geometry over the same region. An advantage of this technique is that it can provide a geographically comprehensive map of the deformation, with a sampling rate far denser than traditional ground surveys e.g. spirit-leveling (Galloway et al., 2007). For each pixel in the interferogram, the phase difference $\left(\phi_{\text {int }}\right)$ is the sum of contributions phases induced by ground surface displacement between tow SAR image acquisitions ( $\phi_{\text {dis }}$ ), topography $\left(\phi_{\text {topo }}\right)$, difference in atmospheric conditions $\left(\phi_{\text {atm }}\right)$, baseline error from imperfect knowledge of orbital or trajectory information $\left(\phi_{\text {orb }}\right)$, and noise $\left(\phi_{\text {noise }}\right)$, as stated in eq. 1

$$
\phi_{\text {int }}=\phi_{\text {dis }}+\phi_{\text {topo }}+\phi_{\text {atm }}+\phi_{\text {orb }}+\phi_{\text {noise }}
$$


In most geophysical applications, the signal of interest is $\phi_{\text {dis }}$. Various techniques are employed to remove other phase terms from the interferogram. The baseline error manifests itself as long wavelength stripes over the interferogram which could be removed using tie points but, the technique cannot be applied if there is a large amount of deformation signal in the interferogram (Hanssen, 2001). However over the test site, there are no severe interseismic/ postseismic signals since it is not situated in an active fault zone and for that the orbital ramps could be possibly detected and removed. Variability in backscattering of radar pulses, system noise and mis-registration results in noise term. In case the application is to detect ground motion, the topographic phase term can be estimated from available DEM (such as SRTM) and removed. Assuming that the wavelength of signal of interest is large enough so that $\phi_{\text {noise }}$ is uncorrelated over the same distance, a common technique to improve signal-tonoise ratio is to multilook or averaging the interferogram with a window (such as $5 \times 5$ ) comparatively small to signal wavelength (Cutberto et al., 2010). Within the averaging window, the signal remains the same while noise tends to cancel out and decreases.

The atmospheric phase term is introduced into the interferogram because of the change in the refractive index of the atmosphere between two acquisitions. Though the atmospheric phase delay depends on several atmospheric factors, Zebker et al. (1997) concluded those atmospheric water vapors are by far the dominant error source in repeat-pass SAR interferometry. For temporal variation, it is known that water vapor values are temporally uncorrelated when their temporal interval is greater than 1 day (Emardson et al., 2003), or even over periods of a few hours, particularly in mountainous areas where air is forced to rise over, or flow around, thereby inducing local overturning and condensation effects at the kilometer scale (Miranda, 1990; Miranda and James, 1992; Webley et al., 2002). The spatial scale of water vapor variability is on kilometer scale (Hanssen et al. 1999), while in Li et al. (2005) it is observed that the atmospheric "ripples" with a characteristic wavelength of 4-12 $\mathrm{km}$. Thus it is correlated within the wavelength of ground displacement and so cannot be removed by any filtering without corrupting the geophysical signal of interest. This is a major problem of 2-pass DInSAR technique especially in tropical areas where the atmospheric phase term is highly expected.

In the same fashion as DEM is utilized to estimate topographic phase term, external data source is required to estimate the atmospheric phase term. Currently, atmospheric water vapor amount has been continuously measured with the two National Aeronautics and Space Administration (NASA) MODIS instruments. The near-IR water vapor products provided by the Moderate Resolution Imaging Spectroradiometer (MODIS) have a spatial resolution of 1 $\mathrm{km} \times 1 \mathrm{~km}$ (at nadir). Even though MODIS data is one of solutions for atmospheric water vapor variation compensation, there is still an important technical problem. As reported by Gao and Kaufman (2003), uncertainties in the surface spectral reflectance, sensor calibration, haze, undetected clouds are estimated to be $5-10 \%$ and errors can be up to $14 \%$ under hazy conditions. The main reason is that MODIS water vapor amounts are derived from the transmittances based on theoretical calculations and using look-up table procedures. However, atmospheric water vapor has very different absorption coefficients over the band passes of MODIS channels centered near 0.935, 0.940, and $0.905 \mu \mathrm{m}$. As a result, the three channels have different water vapor sensitivities under the same atmospheric condition. The strong absorption channel at $0.935 \mu \mathrm{m}$ is most sensitive under dry conditions, while the weak absorption channel at $0.905 \mu \mathrm{m}$ is most sensitive under humid conditions (Gao and Kaufman, 2003). Therefore, at any given atmospheric condition, the derived water vapor values from the three channels can be different. Such that, a mean water vapor value (W) is obtained according to the equation: 


$$
\mathrm{W}=\mathrm{f} 1 \mathrm{~W} 1+\mathrm{f} 2 \mathrm{~W} 2+\mathrm{f} 3 \mathrm{~W} 3
$$

where $\mathrm{W} 1, \mathrm{~W} 2$, and $\mathrm{W} 3$ are water vapor values from the $0.935,0.940$, and $0.915 \mu \mathrm{m}$ channels, respectively, and f1, f2, and f3 are the corresponding weighting functions. Since the mean water vapor value is obtained and this can result in water vapor derivation errors, implying that the calibration of MODIS PWV data is needed before being applied to correct InSAR atmospheric effects.

Li et al. (2003) first presented some results of using MODIS PWV data to correct atmospheric effects on InSAR, one is the MODIS correction model over Mt. Etna. Since there were more pixels closer to zero in an unwrapped image with MODIS correction, implied the MODIS correction was effective. Li et al. (2005) proposed an integration of MODIS PWV data and GPS for InSAR atmospheric correction over the Long Beach-Santa Ana basin, where the GPS data are mainly used to calibrate the MODIS PWV data. The result showed that phase variation of the unwrapped interferogram decreased from 2.48 radians before correction to 1.47 radians after correction. While these results from GPS are encouraging, the unavailability of continuous GPS data over our Bangkok test area leads us to seek alternative source to calibrate MODIS PWV. Qu et al. (2008) reported that the zenith delays calculated using ground meteorological data are in accordance with the GPS observations. It has higher temporal resolution and a better accuracy in term of calculating the zenith delay than MODIS PWV data since being ground observation the presence of clouds or traveling medium has no effect. This research employs ground-based meteorological data to calibrate MODIS PWV.

The investigation on atmospheric phase correction for InSAR begins in section 2 in which details of SAR test images, selection criteria and the formation of interferograms are provided. Section 3 shows procedures of three methods to estimate atmospheric phase terms. The first method is to use MODIS PWV directly to compute the atmospheric phase (Bevis et al., 1992). This will serve as base case upon which the next two methods will measure against. In second method we employ meteorological data to calibrate MODIS PWV before phase computation. Over the test area, the acquisition time of MODIS is 5 hours later than that of TerraSAR-X, the water vapor condition could differ significantly (Li et al., 2009). Third method is an attempt to further improve the second method by estimating the expected MODIS value (acquired around $11 \mathrm{a} . \mathrm{m}$. local time) at the time of the SAR image acquisitions (around 6 a.m.). The results and discussions then follow in section 4. Section 5 presents the main findings and conclusion of the study.

\section{TEST AREA AND DATA}

South-eastern part of Bangkok metropolitan is selected as test area (figure 1). An advantage of this test site is that most of the area are urbanized which means a large portion of stable pixels in SAR images remains coherent over time and thus the phase measurement described in eq. 1 is valid. Situated far from active faults, tectonic deformation can be assumed to be negligible. The main cause of surface deformation is known to be from land subsidence due to over extraction of groundwater (Phienwej et al., 2006). However, the annual rates are known and so can be estimated over the period of repeat SAR acquisitions. Further, the study area is flat with ground elevation gradually decreases from few meters above mean sea level in the north to zero at the coastline. The SRTM DEM that we use to remove topographic phase correspond well with the terrain, which means and the absolute vertical error is much less than $+/-16 \mathrm{~m}$ as stated in its design accuracy (Rabus et al., 2003) and topographic phase terms can be accurately estimated. 

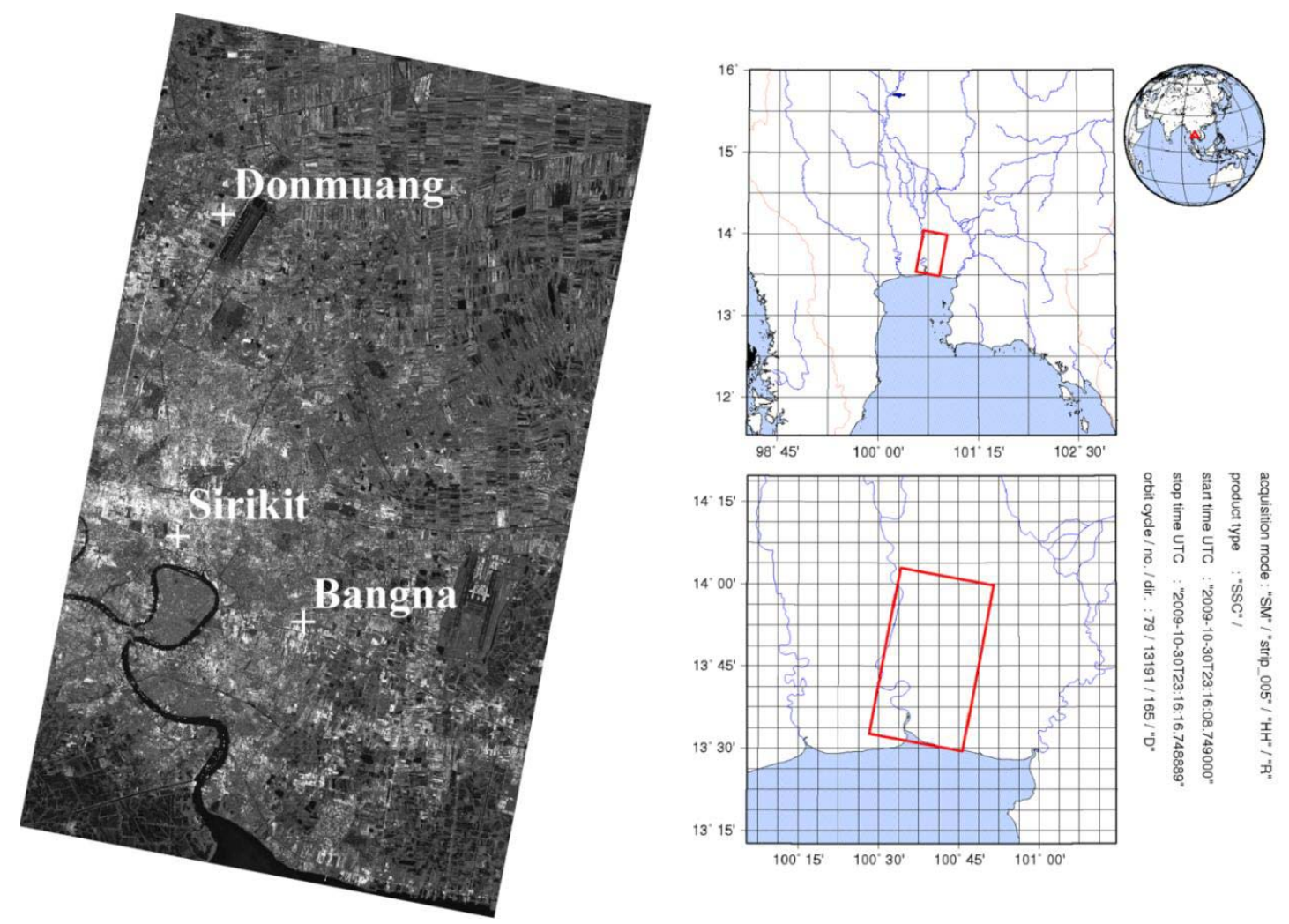

Fig.1. shows TerraSAR-X image track 075 (Descending) acquired on October 30, 2009 over south-eastern Bangkok. Three meteorological stations used in this study are shown; Bangna, Donmuang and Sirikit.

\subsection{IMAGE SELECTION}

Four SAR images were selected from an archive of 23 TerraSAR-X strip-map mode SLC images (descending path, track 075) that had been acquired since September 2009 until June 2010 for the study of Bangkok subsidence. The acquisition in strip-map mode provides SLC images with $3 \mathrm{~m}$ resolution and a spatial coverage of $30 \mathrm{~km}$ x $50 \mathrm{~km}$. The location of study area and the coverage of TerraSAR-X image are shown in figure 1 . As the study aims to correct atmospheric effect in the interferogram, the main selection criterion is that cloud-free MODIS PWV data on the same date of SAR acquisition is available. The criterion can rarely be satisfied in tropical SE Asia where the number of completely cloud-free is very low. We thus chose the four images on the date that we have least cloud-covered MODIS PWV data.

Six interfergrams can be formed by pairing any two of the four chosen TerraSAR-X SLCs. As coherence decreases over time, temporal separation should be kept to minimum in the selection of SLC pairs. Further, the magnitude of deformation phases in the interferogram formed from pairs with small temporal baseline will become small. Over the same area, the maximum subsidence rate during 2005-2009 periods determined from a combined persistent scatterer/small baseline InSAR technique is $15 \mathrm{~mm} / \mathrm{yr}$ (Aobpaet et al., 2010). With this rate, monthly subsidence is less than $1.3 \mathrm{~mm}$ and $\phi_{\text {def }}$ is less than $15^{\circ}(0.26$ radians $)$ significantly less than system noise of $35^{\circ}$ (0.61 radians) as reported in (Poncos et al., 2008). The deformation phase term can then be neglected in the interferogram with less than 1 month temporal baseline. As a result, 2 SLC pairs, with the maximum of 22 -day separation (2 revisit periods), are chosen for the processing to form 2 interferograms for further study. Details of both SLC pairs are shown in table 1. 
Table 1. Shows basic statistics of the 2 interferograms

\begin{tabular}{|c|c|c|c|c|c|c|c|c|}
\hline Int. & Track & Date 1 & Date 2 & days & $\mathrm{B}_{\perp}(\mathrm{m})$ & $\mathrm{B}_{\mathrm{c}}(\mathrm{m})$ & $\mathrm{H}_{\mathrm{A}}(\mathrm{m})$ & $\mathrm{S}$ (radian) \\
\hline 1 & 075 & 30 Oct 09 & 21 Nov 09 & 22 & 42.4 & 3,970 & 213.2 & 0.47 \\
\hline 2 & 075 & 2 Dec 09 & 24 Dec 09 & 22 & 9.1 & 3,970 & 995.1 & 0.10 \\
\hline \multicolumn{8}{|c|}{} \\
$\mathrm{B}_{\perp}=$ Perpendicular baseline (m) \\
$\mathrm{B}_{\mathrm{c}}=$ Critical baseline $(\mathrm{m})$ \\
$\mathrm{H} \mathrm{H}_{\mathrm{A}}=$ Height ambiguity (m) \\
S Phase error due to topographic uncertainty of SRTM (16m) \\
Note: TerraSAR acquisition time is 23:15 UTC or 6:15 local time/ MODIS acquisition time \\
ranges from 10-11 local time
\end{tabular}

\subsection{INTERFEROGRAM FORMATION}

The usual steps for 2-pass DInSAR are image co-registration, interferogram formation, interferogram flattening and removal of the topographic phase term by the use of DEM. Special attention is given to the removal of topographic contributions from interferograms. SRTM DEM is canopy based (Li, 2005), which means it represents a height related to the average phase centre of the radar echoes from scattering elements in the resolution cell such as trees or buildings, not to the ground. While this characteristic may cause problems for other applications required ground surface height, it is advantageous in our case. With large height ambiguity, the $16 \mathrm{~m}$ vertical error of the SRTM DEM results into $26.9^{\circ}$ (0.47 radians) and $5.7^{\circ}(0.10$ radians $)$ in inteferogram 1 and 2 respectively. These phase from DEM error are below the phase noise level of $35^{\circ}$ (0.61 radians) of TerraSAR-X images (Poncos et al., 2008) and so negligible. After removing topographic phase, interferograms are multilooked (2 in rage and 10 in azimuth direction), filtered (5x5 moving average windows), unwrapped, and geocoded to UTM map plane. The remaining phase in the resulted interfergrams (shown in figure $6 \mathrm{a}, 6 \mathrm{e}$ ) is dominated by atmospheric phase terms.

\section{ATMOSPHERIC PHASE TERM}

In this section, an attempt to reduce water vapor effects was made, focusing on the use of independent datasets including MODIS PWV data (figure 2) and ground-based meteorological observations. The Saastamoinen model has been used for tropospheric delay computation in this study using meteorological parameters; relative humidity (RH) and temperature $(\mathrm{T})$, based on the assumption of the linear decrease in temperature with height and the relationship between total pressure and water vapor partial pressure to compute the zenith wet delay (Saastamoinen, 1972). The atmospheric phase term in this study was computed by 3 different methods, the main processing steps of each are shown in figure 3 . The first method (referred to hereafter as method 1) is to use MODIS PWV directly to compute the atmospheric phase (Bevis et al., 1992). The second method we employ meteorological data to calibrate MODIS PWV before phase computation while in the third method, an attempt to further improve the second method by estimating the expected MODIS value at the time of the SAR image acquisitions was made. Processing details on each method are as follow. 

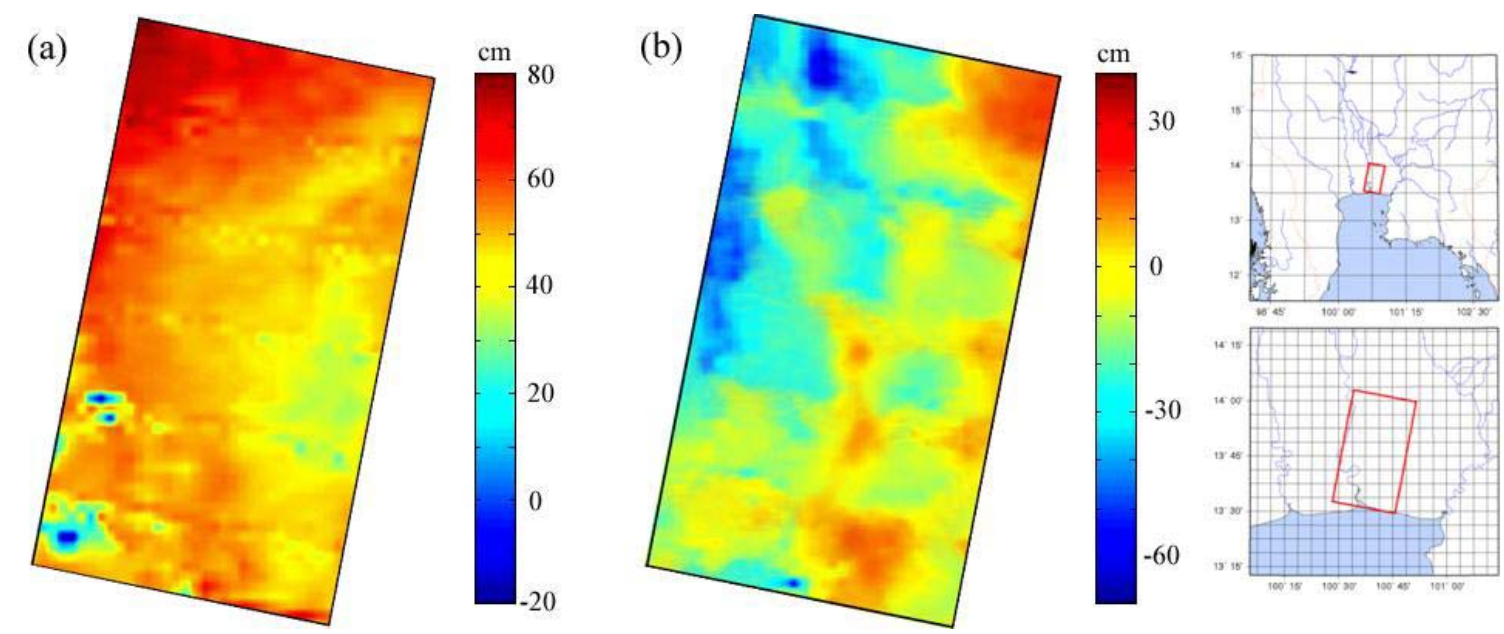

Fig.2. Show the differential MODIS PWV data used in this study (a) 30 October $2009-21$ November 2009, (b) 2 December 2009 - 24 December 2009

\subsection{METHOD 1}

MODIS PWV data with the least cloud-covered were selected since there was no completely cloud-free data within the test area during the TerraSAR-X acquisitions. Satellites scan line errors which values were significantly different in variance and mean of adjacent lines were masked out while the bi-cubic polynomial interpolation method was used to fill in the missing values. Then, PWV data was converted into ZWD using conversion factor using the following ratio:

$$
Z W D=\Pi \times P W V
$$

where $\Pi$ is a conversion factor which is dimensionless and usually ranges from 6.0 to 6.5 . An average conversion factor of 6.2 is adopted in this research. It should be noted that since MODIS is a passive tool (one-way acquisition mode) while SAR instrument is the active tool (two-way acquisition mode), the ZWD data were calculated with double value. In order to align MODIS data with the SAR image for the pixel-by-pixel phase correction, the image-toimage co-registration was performed based on the external DEM, in this case using the SRTM DEM as a reference. After that by using the simplest mapping function $1 / \sin (\xi)$ when $\xi$ is the elevation angle, zenith wet delays are converted into slant path delays. With the varying range of TerraSAR-X elevation angle between far range $\left(65^{\circ}\right)$ and near range $\left(62^{\circ}\right)$. Finally, MODIS water vapor data in slant path delay was divided by TerraSAR-X band wavelength $(31 \mathrm{~mm})$ to convert into phase.

\subsection{METHOD 2}

In method 2 we employ meteorological data to calibrate MODIS PWV before phase computation. Following the processing step in method 1 after applied the conversion factor, at this point before the image co-registration, the second method diverted from the usual processing sequence with the insertion of a calibration process. Following the calibration approach made by Li et al. (2005) where the GPS data were used to calibrate the MODIS data. As reported by $\mathrm{Qu}$ et al. (2008) that the zenith delays calculated using ground meteorological data are also in accordance with the GPS observations. In this research, ground-based meteorological data is used for calibration purpose due to the unavailability of continuous GPS data over Bangkok test area. The Saastamoinen model has been used for 
zenith wet delay computation in this study since it is a good and accurate method to compute zenith wet delay if surface meteorological data are available (Katsougiannopoulos et al., 2006) and in general better than the others (Kos, 2008) with an estimated accuracy of $3 \mathrm{~cm}$ in zenith (Mendes, 1999). Based on the assumption of the linear decrease in temperature with height and the relationship between total pressure and water vapor partial pressure, ZWD can be computed using the following equation (Saastamoinen, 1972):

$$
Z W D=0.002277\left(\frac{1255}{T}+0.05\right) e_{0}
$$

where $\mathrm{T}$ is surface temperature in degrees Kelvin. $e_{0}$ is surface water vapor partial pressure in $\mathrm{hPa}$ and can be derived from the relative humidity $\mathrm{RH}[\%]$ using:

$$
e_{0}=\frac{R H}{100 e^{\left(-37.2465+0.213166 T-0.000256908 T^{2}\right)}}
$$

As such, the meteorological data covering the scanning time of MODIS acquisition were then selected and compared with MODIS ZWD data under clear sky conditions during a 2 year-period ranging from December 2008 to February 2010, assuming the relationship between ZWD to be linear:

$$
Z W D_{S A A S}=a \times Z W D_{M O D I S}+b
$$

where $Z_{W D D}$ is the zenith wet delay computed from the Saastamoinen model at the time of MODIS acquisition while the $\mathrm{ZWD}_{M O D I S}$ is zenith wet delay computed from MODIS water vapor data. After that, a scale factor (a) computed from each station was spatially interpolated with Inverse Distance Weighting (IDW) method before applied to MODIS data. It should be noted that only $\mathrm{ZWD}_{M O D I S}$ values within $2 \sigma$ are brought into comparisons, to which errors can come from some cloudy pixels which are falsely identified as cloud free. Regression results are shown in the left column of figure 4.

\subsection{METHOD 3}

Since there are only time intervals of up to 60 minutes between MODIS and ERS data used in Li's experiment, while it is much wider of time interval up to 5 hours difference with the TerraSAR data. As reported by Emardson et al. (2003), that water vapor values are temporally uncorrelated when their temporal interval is greater than 1 day. Such that, the water vapor condition could differ significantly. In method 3 , there is an attempt to improve method 2 by estimating the expected MODIS value (acquired around $11 \mathrm{a} . \mathrm{m}$. local time) at the time of the SAR image acquisition (around $6 \mathrm{a} . \mathrm{m}$.). Additional to the calibration approach of method 2 but this time, ZWD computed from the meteorological data covering the scanning time of MODIS measurement were compared with the meteorological data covering the scanning time of TerraSAR-X during the same 2 year-period, assuming the relationship between ZWD to be linear:

$$
Z W D_{\text {TerraSAR }}=a \times Z W D_{M O D I S}+b
$$

where $Z_{W D} D_{\text {TerraSAR }}$ is the zenith wet delay computed from the Saastamoinen model at the time of TerraSAR acquisition while the $\mathrm{ZWD}_{M O D I S}$ is the zenith wet delay computed from the 
Saastamoinen model at the time of MODIS acquisition. After that, a scale factor (a) computed from each station was spatially interpolated with IDW interpolation method and combined with the interpolated map resulted from method 2 to make a time-different calibration map before applied to MODIS data. Regression results are shown in the right column of figure 4.

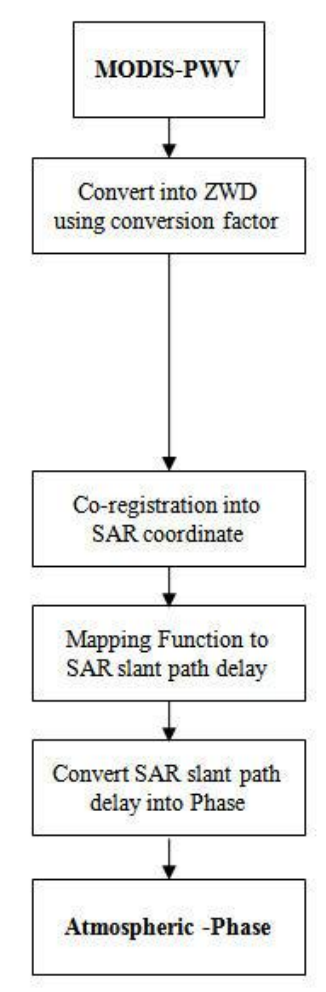

Method 1

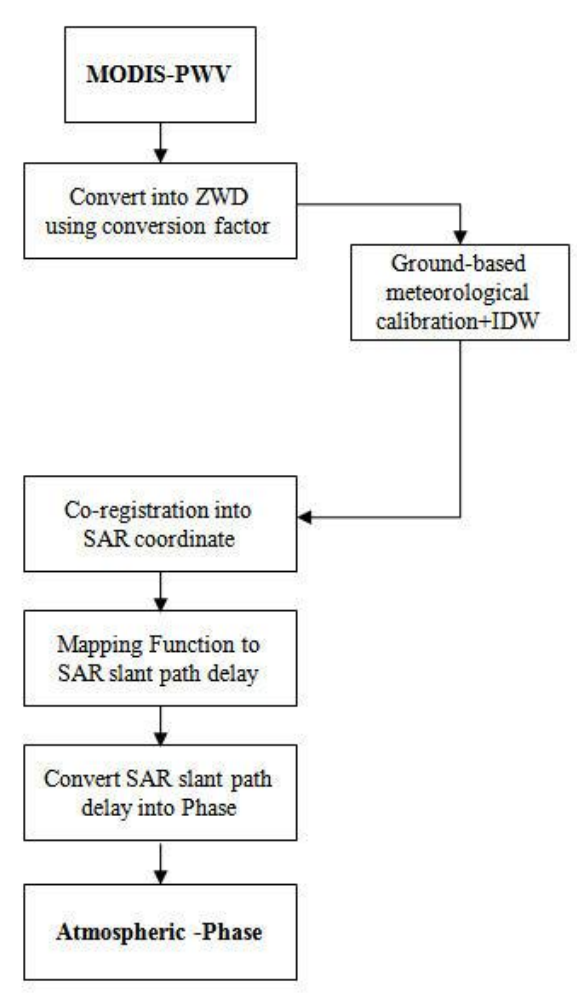

Method 2

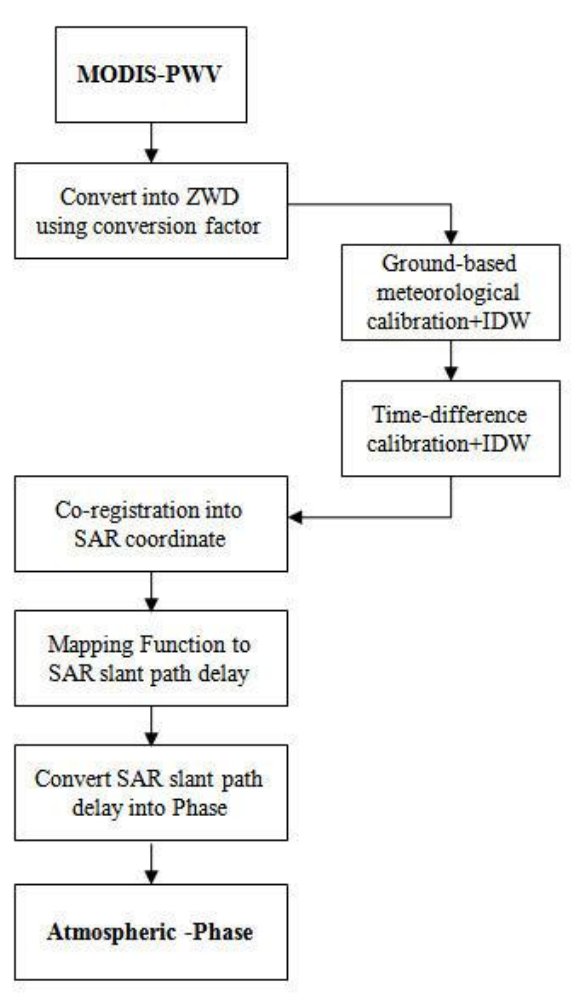

Method 3

Fig.3. Show processing flow charts of how to convert the MODIS PWV data into phase in 3 different methods.

\section{RESULTS AND DISCUSSIONS}

The calibration result using ground-based meteorological data indicated that $\mathrm{ZWD}_{M O D I S}$ was almost identical with $Z \mathrm{WD}_{S A A S}$ at Bangna station by an indifferent scale factor of 1.007, while at Donmuang station; it indicated that $\mathrm{ZWD}_{M O D I S}$ was larger by a scale factor of 1.208 . In contrast with Sirikit station, it was found to be smaller than $\mathrm{ZWD}_{S A A S}$ by a scale factor of 0.916 . The number of valid samples was 49,50 and 33 respectively, for roughly $25 \%$ of all MODIS PWV observations appear to be cloud free on each meteorological station. The calibration for the time-difference between $\mathrm{ZWD}_{M O D I S}$ and $\mathrm{ZWD}_{\text {TerraSAR }}$ at Bangna, Donmuang and Sirikit meteorological station indicated that $Z \mathrm{ZWD}_{\text {TerraSAR }}$ was smaller than that of $\mathrm{ZWD}_{M O D I S}$ with the scale factor of $0.923,0.886$ and 0.924 respectively. In term of the calibration results, it shows that there are good linear relationships between $Z_{W D} W_{M O D I S}$ and the $\mathrm{ZWD}_{S A A S}$ and also $\mathrm{ZWD}_{M O D I S}$ with the $\mathrm{ZWD}_{\text {TerraSAR }}$.

The TerraSAR-X interferogram-1 (October 30, 2009 - November 21, 2009) and 3 different atmospheric-corrected scenarios over Bangkok are shown in figure $6 \mathrm{a}, 6 \mathrm{~b}, 6 \mathrm{c}, 6 \mathrm{~d}$. It is observed that correction method 3 (figure 6d) provides the best result since the large phase 
delays (shown in brown color) around 30-40 mm were largely removed, leaving lower phase delay value in the corrected interferogram. In figure $5 \mathrm{a}, 5 \mathrm{~b}, 5 \mathrm{c}, 5 \mathrm{~d}$, the histogram plots of the interferogram and its atmospheric corrected versions shows bi-modal distribution. It could be clearly seen that pixels of interferogram corrected by method 3 has the main peak near zero and the other peak at around 20, both of which move towards the ideal pixel value of zero. However, a small percentage of pixels, especially those in the south-eastern part area, are over-corrected. Overall results show that the correction method 1 and 2 are indifferent while method 3 gives better results with all the lower statistic values such as mean, mode except that of RMSE which is similar to the other two methods. Histogram of correction method 1 and 2 also give a much higher number of pixels in lower range of phase delay values (around 0-20 mm) when compared with the non-corrected interferogram.
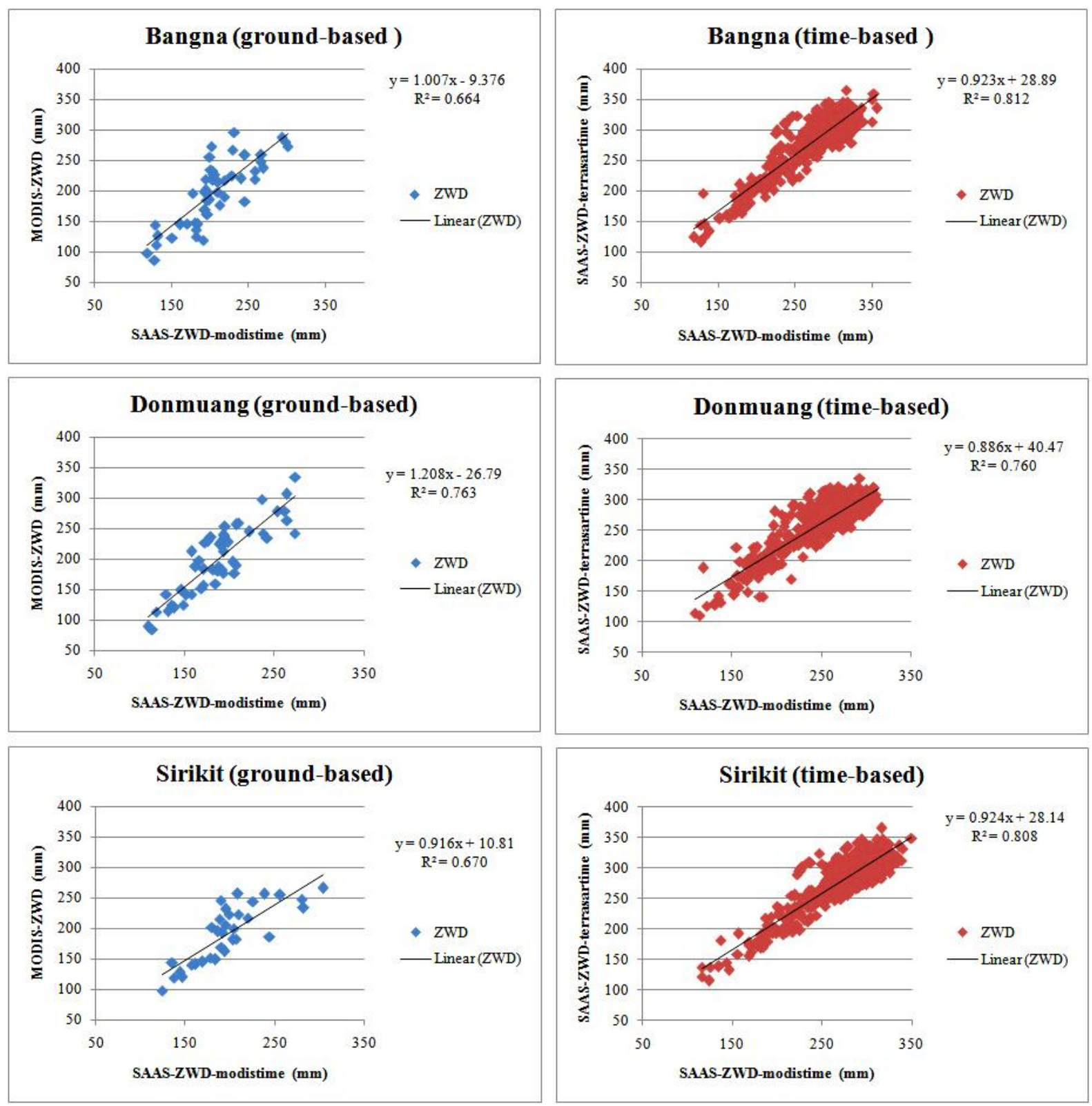

Fig.4. Show results of the calibration 1 and 2 of 3 meteorological stations namely; Bangna, Donmuang and Sirikit. Geographical location of the 3 stations can be seen in figure 1. 
Statistics of phase delay are validated with two different methods. Although the phase delays using the latter method are lowered in case of figure $6 \mathrm{~d}$ but, the phase pattern has also changed a lot with fluctuations and this might arguably be concluded a better correction results. Consider the two methods, the first method looks for the smallest error (variation) or the RMSE. Since the RMSE is based on averaging values, a drawback of the method is that it can be dominated with few but really large terms which can mislead the true value. More importantly, the values might really not indicate how closer the correction result to the expected value. The second method on the other hand, measures the number of corrected pixels even though sometimes the overall correction result left with large errors which cause a higher RMSE. If the goal of error estimation is to provide an estimate that is close to the expected value. The closeness is better measured by counting pixels directly. As such, even though the second method may have larger RMSE but a higher probability close to the expected value and should be chosen for the validation. As indicated by the peak of the histogram which moves towards zero which means the success rate is higher.

The results from interferogram-2 (2 December 2009 - 24 December 2009) are shown in figure $6 \mathrm{e}, 6 \mathrm{f}, 6 \mathrm{~g}, 6 \mathrm{~h}$ and the histogram plots are shown in figure $5 \mathrm{e}, 5 \mathrm{f}, 5 \mathrm{~g}, 5 \mathrm{~h}$. The correction patterns are similar which the phase delays in dark yellow color around 10-20 mm were largely removed in the north-eastern part and the center area of the interferogram-2 (figure $6 \mathrm{~b}$, $6 \mathrm{c}, 6 \mathrm{~d})$. The main improvement from applying atmospheric correction is that more pixels are moved towards the ideal value of zero, resulting in higher pixel numbers of low values and smaller count of high values. However, certain pixels have larger delays as unlike the case of interferogram-1; however, no significant difference between different correction methods is observed. As all statistics such as mean, mode and RMSE are similar in all cases (table 2). It could also be noted that RMSE is not a sufficient indicator if phases in the interferogram are less fluctuated than the water vapor data which results in a higher RMSE even though there are more pixels with lower delay value within the interferogram after the correction.

Overall correction results show that, with the calibration of MODIS data gives better results when compared with the non-calibrated one. When created the differential map between the non-correction one and each correction method. Correction with method 3 gives the best results when compared with the increasing amount of lower delay pixels (table 3), but, also it gives the most errors (negative pixels) from over-correction (figure $6 \mathrm{~d}$ ). The method 1 and 2 however, almost give indifferent results which is conflicting with what reported in Li et al. (2005). This may due to the number of reference stations used in which in Li's number of GPS stations vary from 72-90 or an average of 80 over the SCIGN region which covers the area around $22,500 \mathrm{~km}^{2}(\mathrm{Li}, 2004)$ or the density of 1 GPS station per 280 $\mathrm{km}^{2}$ compared to only 3 meteorological stations within $1,500 \mathrm{~km}^{2}$ or 1 station per $500 \mathrm{~km}^{2}$ in this research.

One possible reason which gives the correction results in interferogram-1 better than interferogram-2 is the different in spatial variation of MODIS-PWV data (figure 2). Since both interferogram after the topographic phase removal produced a relatively smooth atmospheric phase variation. With a spatial smoothness of MODIS water vapor data similar to that spatial smoothness of atmospheric phase in the interferogram-1, the overall results were better. Also, the IDW interpolation which producing a smooth surface, tend to have an effect with the smooth surface more than the fluctuated one, in this case it benefit more for MODIS data applied for interfergram-1 with a smoother surface. When compare between methods, which the correction result in method 3 was better significantly in term of phase delay reduction. It is found that the calculation of phase delay differs significantly (from $\pi$ to $-\pi$ ); even from a slightly change of water vapor data if within a smooth surface. 
Table 2. shows results statistics of phase delay in mm. of both interferogram with 3 corrected scenarios. Statistics are calculated within a coherence threshold of $>0.5$.

\begin{tabular}{|l|r|r|r|r|r|}
\hline Interferogram-1 & Min & Max & Mean & Mode & RMSE \\
\hline non-correction & -34.5 & 78.5 & 26.2 & 22.6 & 13.5 \\
\hline method 1 & -34.6 & 86.0 & 23.7 & 18.3 & 11.0 \\
\hline method 2 & -32.0 & 73.6 & 22.9 & 18.6 & 10.7 \\
\hline method 3 & -72.0 & 61.7 & 5.9 & 6.0 & 11.1 \\
\hline Interferogram-2 & Min & Max & Mean & Mode & RMSE \\
\hline non-correction & -28.2 & 79.3 & 18.3 & 12.5 & 8.6 \\
\hline method 1 & -61.4 & 76.2 & 15.7 & 10.6 & 10.3 \\
\hline method 2 & -62.2 & 79.5 & 15.7 & 15.8 & 10.6 \\
\hline method 3 & -76.2 & 79.5 & 15.1 & 10.0 & 10.1 \\
\hline
\end{tabular}

Table 3. shows results statistics of pixels in each correction method after differentiated with the non-corrected interferogram. The pixels in differential map which gives value $>0$ mean the method lowered the phase delay or improve the interferogram, while pixels with gives value $<0$ mean the method added more delay (errors) to the original interferogram.

Statistics are calculated within a coherence threshold of $>0.5$.

\begin{tabular}{|l|r|r|r|r|}
\hline Interferogram-1 & Lower delay & Indifference & Higher delay & total pixel \\
\hline method 1 & 452,556 & 3,861 & 181,314 & 637,731 \\
\hline method 2 & 478,454 & 3,796 & 155,481 & 637,731 \\
\hline method 3 & 634,833 & 69 & 2,829 & 637,731 \\
\hline Interferogram-2 & Lower delay & Indifference & Higher delay & total pixel \\
\hline method 1 & 412,321 & 2,253 & 351,365 & 765,939 \\
\hline method 2 & 438,517 & 2,311 & 325,111 & 765,939 \\
\hline method 3 & 431,493 & 2,786 & 331,660 & 765,939 \\
\hline
\end{tabular}

\section{CONCLUSIONS}

This study has shown that estimation of phase delays from MODIS is improved by calibrating them with ground meteorological data. The study has shown that calibration results shows that there are good linear relationships between MODIS-ZWD and the Saastamoinen modelZWD even though the MODIS-ZWD appeared to be overestimated with the ones computed from the model. Since the ZWD estimated from these two sources were consistent thus, it is proposed to integrate Saastamoinen model and MODIS data for InSAR atmospheric correction. In this research, it was found that water vapor data with less spatial fluctuation give better atmospheric correction results. The 5 hour time-shifted linear fit models along with the IDW interpolation showed that there is significant improvement on atmospheric phase delay correction using MODIS water vapor product.

There has been a need for surface deformation study to remove atmospheric phase term from interferograms created by 2-pass DInSAR technique. This is especially the case when the area of interest is in tropical zone and the assumption adopted in many studies of arid or semi-desert areas that the atmosphereic phase term due to water vapor content is small comparing with the deformation signal may not be valid. The technique presented in this paper can be easily adopted for the estimation of phase delay. As the availability of cloud-free MODIS data is limited, focus on further investigation is on the derivation of phase delay without MODIS from combination of GPS and meteorological data and the required combined density of which that can provide reliable estimate of atmospheric phase delay. 

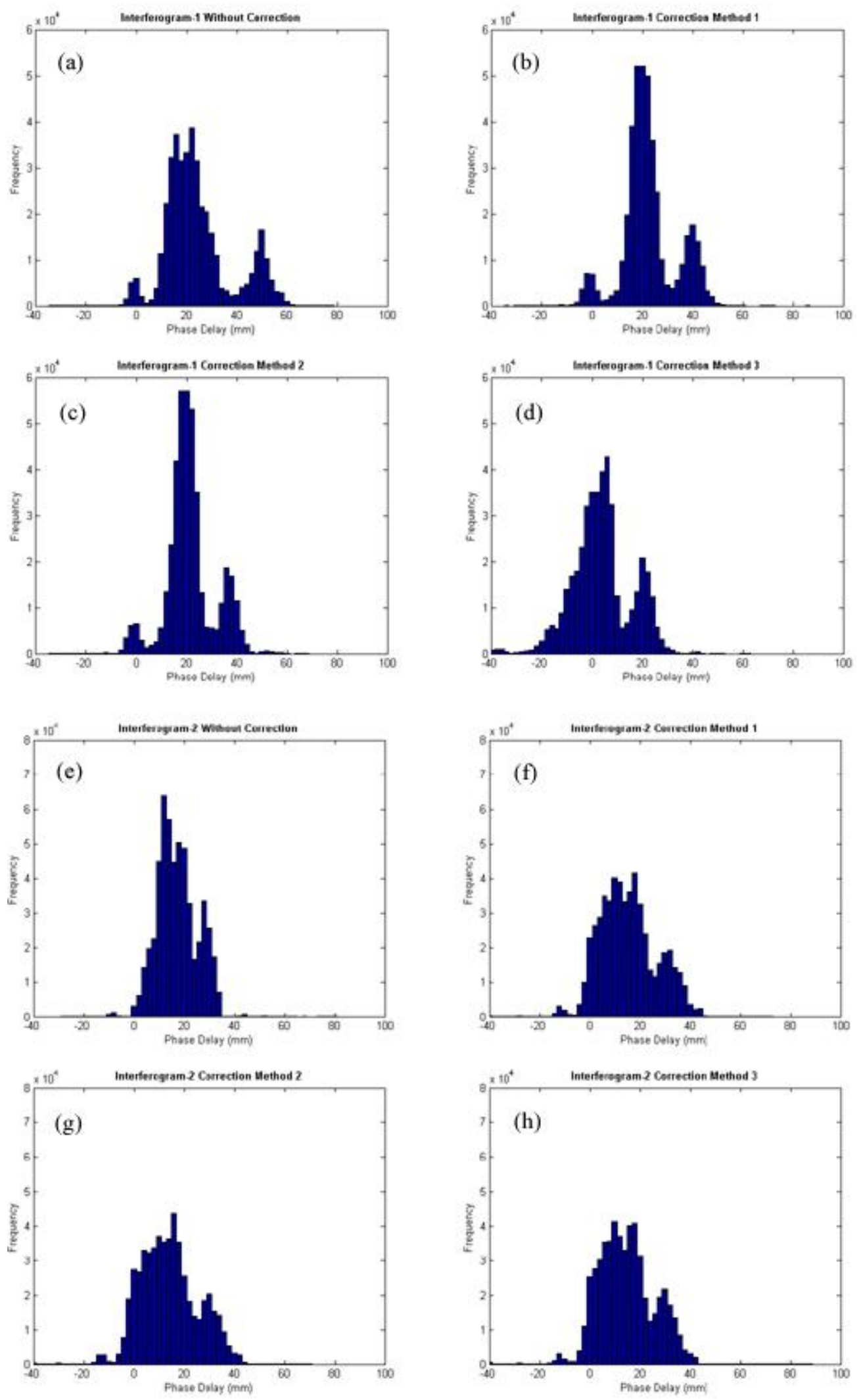

Fig. 5a., 5b., 5c., 5d. Show histogram of interferogram-1 consists of the interferogram without atmospheric correction and three corrected scenarios using Method 1, Method 2 and Method 3 respectively while Fig. 5e., 5f., 5g., 5h. Show histogram of the interferogram-2 arranging in the same order as in interferogram-1. Pixels plotted in all histogram are selected within a coherence threshold of $>0.5$. 


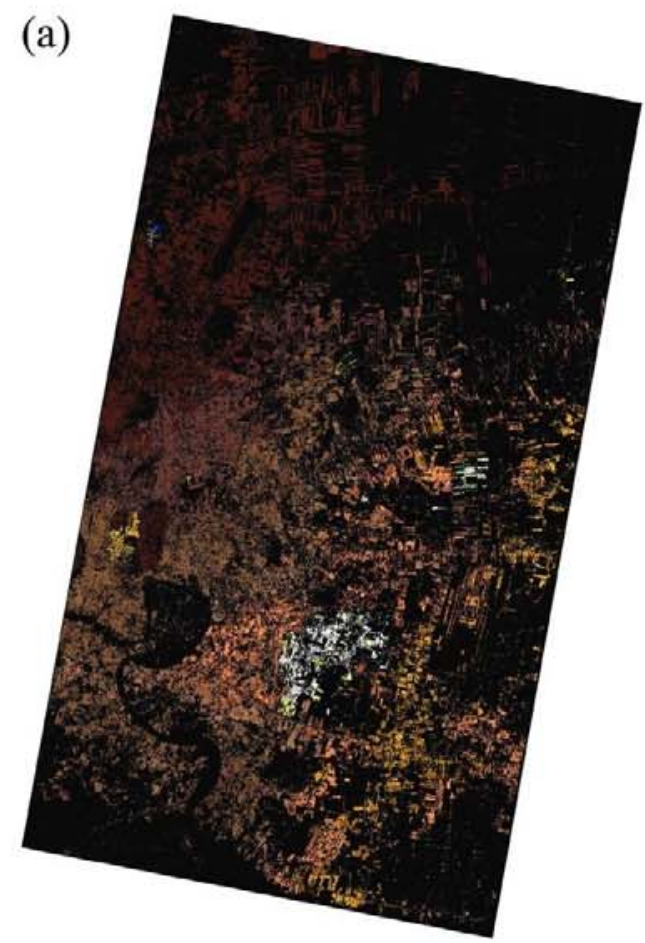

Unwrapped Interferogram-1

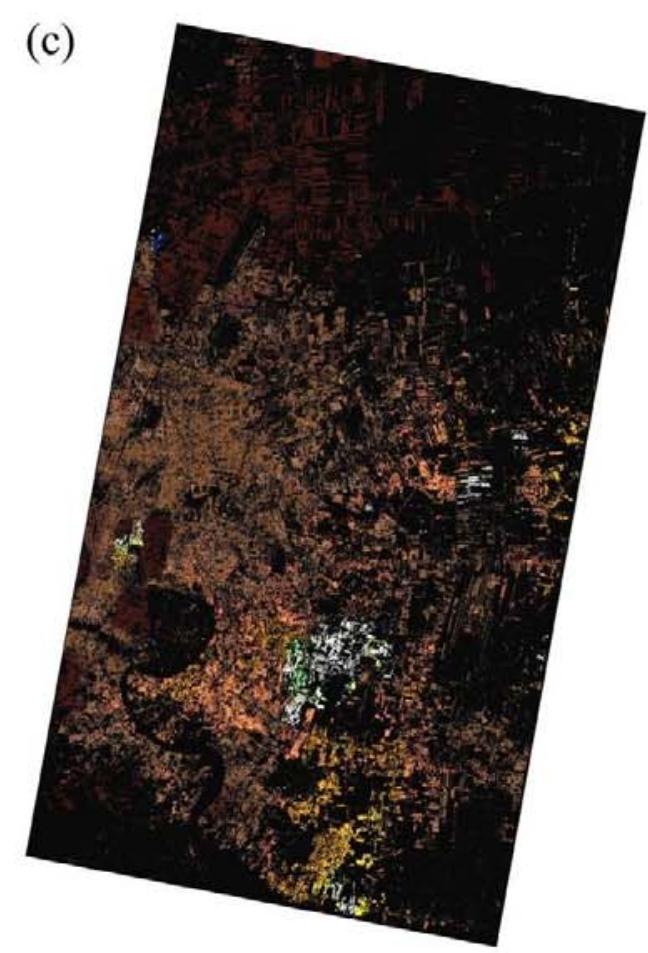

Correction Method 2

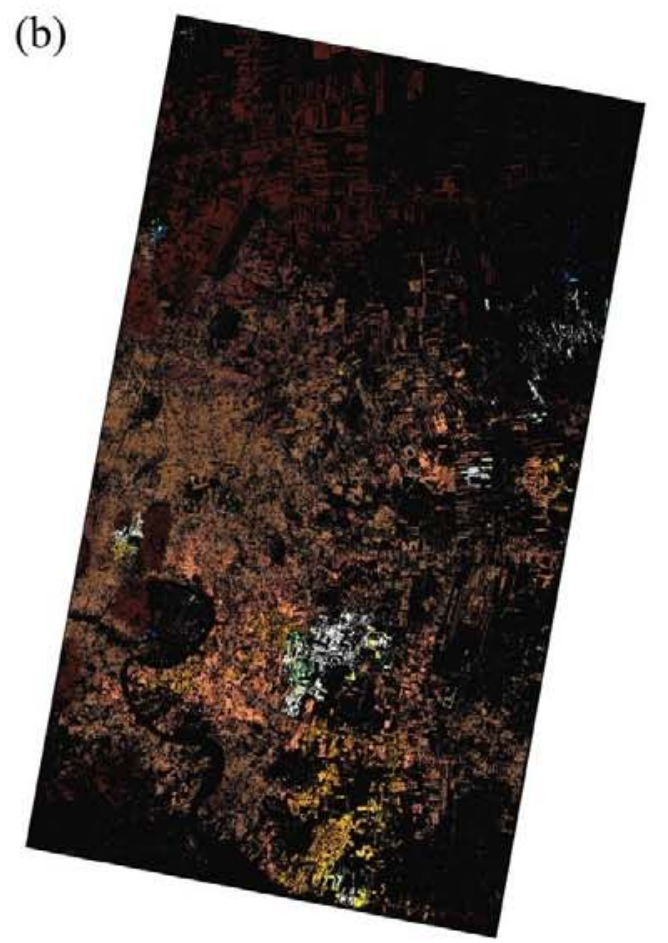

Correction Method 1

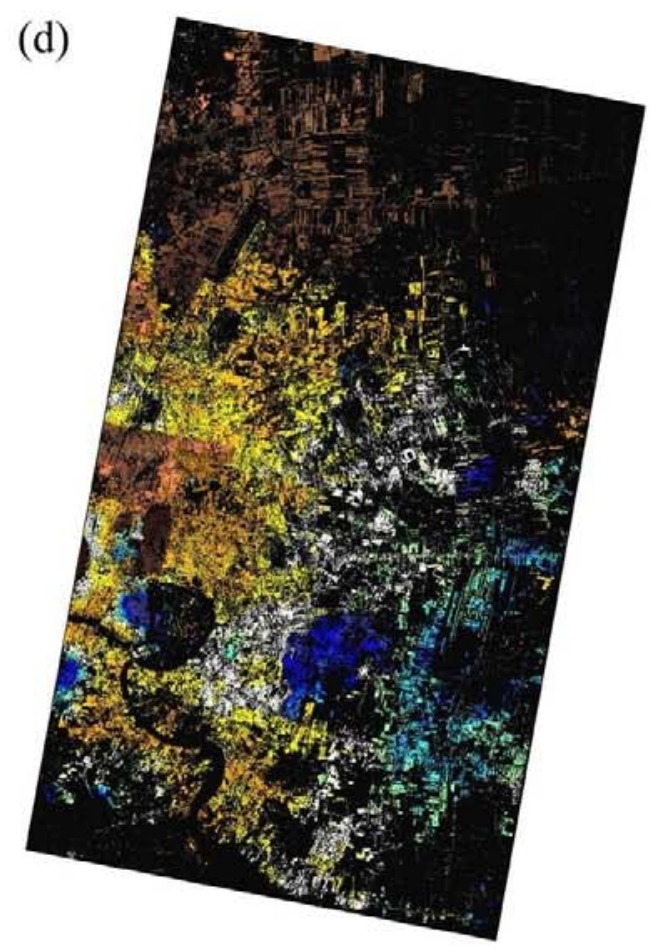

Correction Method 3

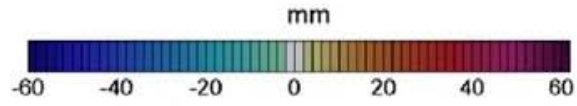

Fig. 6a., 6b., 6c., 6d. Show unwrapped interferogram-1 (30 October 2009 - 21 November 2009) and 3 correction scenarios, only pixels with a coherence threshold of $>0.5$ were considered in phase unwrapping. 


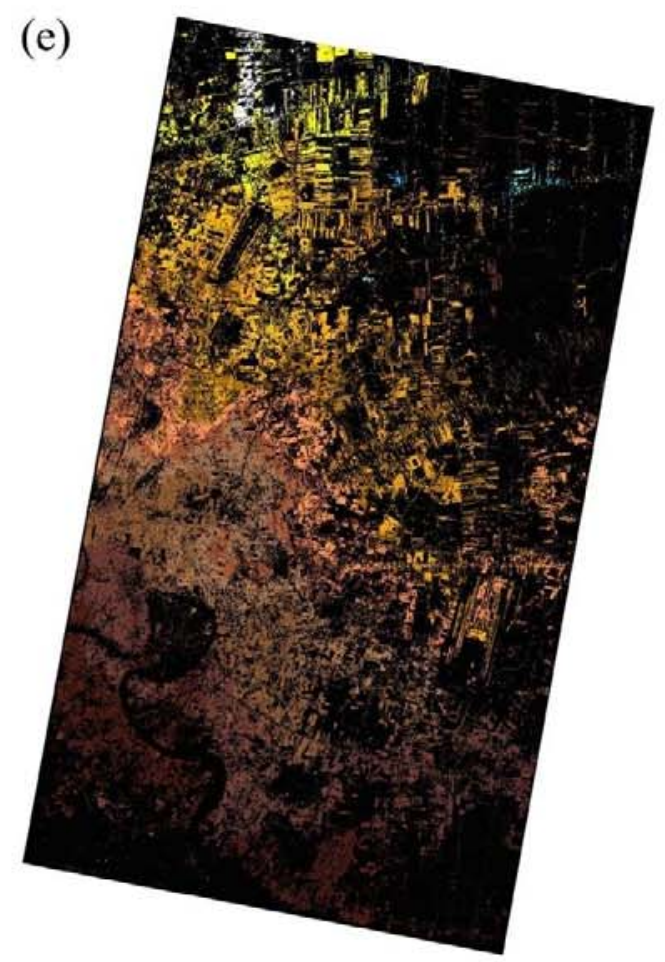

Unwrapped Interferogram-1

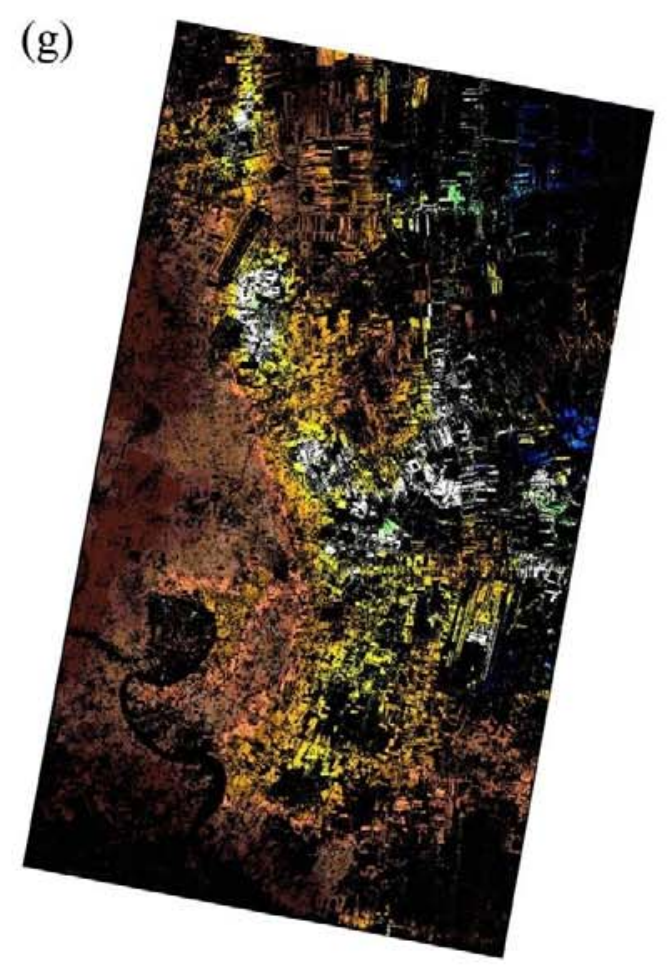

Correction Method 2

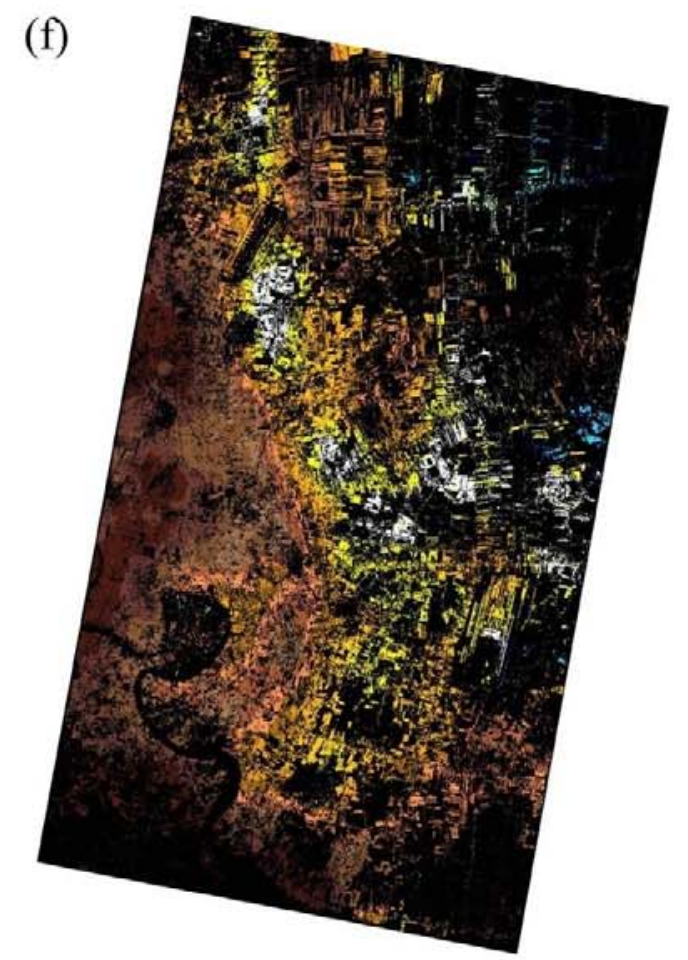

Correction Method 1

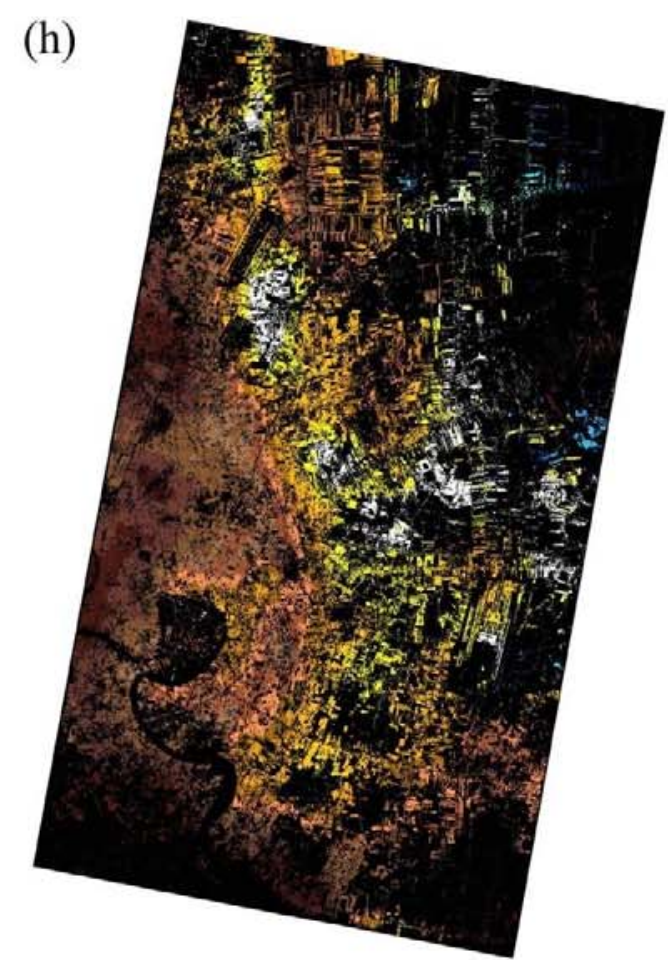

Correction Method 3

$\mathrm{mm}$

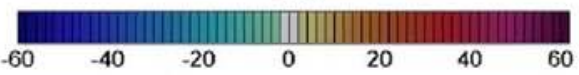

Fig. 6e., 6f., 6g., 6h. Show unwrapped interferogram-2 (2 December 2009 - 24 December 2009), same scenarios were shown as interferogram-1. 
Acknowledgements. TerraSAR-X images used in this study is provided by DLR through a Thailand-EU joint research: Earth Observation Technologies for Thailand: Environmental Change Detection and Investigation (GEO2TECDI) program. MODIS data are freely downloaded from http://modis.gsfc.nasa.gov/; SRTM DEM is freely downloaded from http://www2.jpl.nasa.gov/srtm/cbanddataproducts.html. The authors would like to thank Meteorological Department of Thailand for making the ground meteorological data available for our study.

\section{REFERENCES}

Aobpaet, A., Cuenca, M., Hooper, A., Trisirisatayawong, I. (2010). Land subsidence evaluation using InSAR time series analysis in Bangkok metropolitan area. Fringe 2009 Workshop. Frascati, Italy.

Bevis, M., Businger, S., Herring, T.A., Rocken, C., Anthes, R.A., \& Ware, R. H. (1992). GPS meteorology: remote sensing of atmospheric water vapor using the Global Positioning System. Journal of Geophysical Research, 97(D14), 15, 787-715, 801.

Cutberto, U. P., \& Wilver, E. S. (2010). An accuracy assessment of spaceborne x-band (TerraSAR-X) spotlight mode InSAR DEMs. Accuracy 2010 Symposium. Leicester, UK.

Emardson, T., Simons, M., \& Webb, F. (2003). Neutral atmospheric delay in interferometric sysnthetic aperture radar applications: Statistical description and mitigation. Journal of Geophysical Research, 108, 2231.

Galloway, D., \& Hoffman, J. (2007). The application of satellite differential SAR interferometry-derived ground displacements in hydrogeology. Hydrogeology Journal , 15 (1), 133-154.

Gao, B. C., \& Kaufman, Y. (2003). Water vapor retrievals using Moderate Resolution Imaging Spectroradiometer (MODIS) near-infrared channels. Journal of Geophysical Research , 108 (D13, 4389).

Hanssen, R. F., Weckwerth, T. M., Zebker, H. A., \& Klees, R. (1999). High-resolution water vapor mapping from interferometric radar measurements. Science, 283 (5406), 12971299.

Hanssen, R. (2001). Radar Interferometry: Data interpretation and Error Analysis. Kluwer Academic Publishers: Dordrecht.

Katsougiannopoulos, S., Pikridas, C., Rossikopoulos, D., Ifadis, I. M., \& Fotiou, a. A. (2006). Tropospheric Refraction Estimation Using Various Models, Radiosonde Measurements and Permanent GPS data. XXIII FIG Congress. Munich, Germany.

Kos, T., Botincan, M., \& Markezic, I. (2008). Estimation of Tropospheric Delay Models compliance. 50th International Symposium ELMAR-2008. Zadar, Croatia.

Li, Z., Muller, J., Cross, P. (2003). Tropospheric correction techniques in repeat-pass SAR interferometry. Proceedings of the Fringe 2003 workshop, ESA ESRIN. Frascati, Italy.

Li, Z. (2004). Production of regional $1 \mathrm{~km} \mathrm{x} 1 \mathrm{~km}$ water vapor fields through the integration of GPS and MODIS data. Proceedings of the 17th International Technical Meeting of the Satellite Division of The Institute of Navigation. Long Beach, California, USA.

Li, Z., Muller, J., Cross, P., \& Fielding, E. J. (2005). Interferometric synthetic aperture radar (InSAR) atmospheric correction: GPS, Moderate Resolution Imaging Spectroradiometer (MODIS), and InSAR integration. Journal of Geophysical Research , 110. 
Li, Z., Fielding, E.J., Cross, P., \& Preusker, R. (2009). Advanced InSAR atmospheric correction: MERIS/MODIS combination and stacked water vapour models. International Journal of Remote Sensing , 30(13), 3343-3363.

Mendes, V. (1999). Modeling the neutral-atmosphere propagation delay in radiometric space techniques. PhD thesis. New Brunswick, Canada: University of New Brunswick.

Phienwej, N. G. (2006). Bangkok Land Subsidence. Engineering Geology , 82 (4), 187-201.

Poncos, V., \& Dana, I. (2008). Interferometric Generation of Digital Elevation Models for Urban Areas Using TerraSAR-X.

Qu, W. J., Zhu, W.-Y., Song, S. L., \& Ping, J. S. (2008). Evaluation of the Precision of Three Tropospheric Delay Correction Models. Chinese Astronomy and Astrophysics , 32 (4), 429-438.

Rabus, B. E. (2003). The shuttle radar topography mission - a new class of digital elevation models acquired by spaceborne radar. Journal of Photogrammetry and Remote Sensing , 241-262.

Saastamoinen, J. (1972). Atmospheric correction for the troposphere and stratosphere in radio ranging of satellites. Geophysics monograph 15, 3rd Int. Symp. Use of Artificial Satellites for Geodesy, $A G U$, (pp. 247-251).

Webley, P. B. (2002). Atmospheric water vapour correction to InSAR surface motion measurements on mountains: results from a dense GPS network on Mount Etna. Phys. Chem. Earth, 363-370.

Zebker, H. A., Rosen, P. A., \& Hensley, S. (1997). Atmospheric effects in interferometric synthetic aperture radar surface deformation and topographic maps. Journal of Geophysical Research, 102, 7547-7563.

Received: 2011-08-26,

Reviewed: 2011-10-06, by Zhenhong Li,

Accepted: 2011-10-28. 